\title{
Adapting to Crisis: A Binational Study on the Impact of Flexibility on Latent Profiles of Coping with COVID-19
}

\author{
Alla Hemi ${ }^{1 *}$, M. Roxanne Sopp ${ }^{1,2 *}$, Sarah K. Schäfer ${ }^{3}$, Tanja Michael ${ }^{2}$, Einat Levy-Gigi ${ }^{1,4}$ \\ * These authors contributed equally to this work \\ ${ }^{1}$ Faculty of Education, Bar Ilan University, Israel \\ ${ }^{2}$ Division of Clinical Psychology and Psychotherapy, Department of Psychology, Campus \\ A1 3, Saarland University, D-66123 Saarbrücken, Germany \\ ${ }^{3}$ Leibniz Institute for Resilience Research (LIR), Germany \\ ${ }^{4}$ The Gonda Multidisciplinary Brain Research Center, Bar Ilan University, Israel
}

We have no conflict of interest to disclose.

The data of this study is available at

https://osf.io/v8kjm/?view_only=9648976151874ae9ad04d628cfff7ed4

Correspondence concerning this article should be addressed to Einat Levy-Gigi, Faculty of Education and the Multidisciplinary Brain Research Center, Bar Ilan University, Ramat Gan, Israel.

Email: Einat.Levy-gigi@biu.ac.il 


\begin{abstract}
Introduction. The high level of uncertainty brought by the COVID-19 pandemic has affected the general population's well-being and ability to cope with daily challenges. Studies indicate that flexibility, defined as the ability to employ and adapt a variety of emotional, cognitive, and behavioral strategies in accordance with changing contextual demands, may significantly contribute to coping with long-term stressors such as COVID-19. Objectives. We aimed to investigate which facets of flexibility predict different latent coping profiles in Israel and Germany. Methods. 2330 Israelis and 743 Germans completed online questionnaires measuring cognitive, emotional and trauma-related regulatory flexibility, and cognitive, emotional, and behavioral coping with COVID-19. Results. Analyses revealed three distinct coping profiles in each country (high, medium and low). These profiles differed in both anxiety and depression symptoms with the low coping group experiencing clinically relevant symptoms both in Israel and in Germany. Additionally, cognitive flexibility and trauma-related regulatory flexibility, but not emotion regulatory flexibility, emerged as significant predictors of coping in both countries. Conclusions. Training cognitive and trauma-related regulatory flexibility may help individuals to better cope with psychosocial stressors such as COVID-19. Such trainings could be selectively administered to less flexible subpopulations, as well as adapted to the specific population characteristics.
\end{abstract}




\section{Adapting to Crisis: A Binational Study on the Impact of Flexibility on Latent Profiles of Coping with COVID-19}

The worldwide spread of the Coronavirus disease (COVID-19) in March 2020 has drastically changed everyday lives across the globe (Goldfarb, 2020). These changes and the high level of uncertainty that came with them have affected the general population's wellbeing and ability to cope with daily challenges (Paredes et al., 2021). As a result, a significant rate of the general population has been experiencing mental health problems (Pfefferbaum \& North, 2020). Current meta-analyses indicate that $33 \%$ has experienced anxiety whereas $30 \%$ has experienced depression (Salari et al., 2020; Wang et al., 2020). Since these estimates are based on self-report data rather than confirmed diagnoses, they cannot be used as indicators of disease prevalence. Nevertheless, they illustrate a significant momentaneous impact of COVID-19 on mental health, as also evident in high rates of sleep problems (34\%) and general stress (36\%) (Krishnamoorthy et al., 2020). Critically, these rates mostly come from cross-sectional studies investigating acute responses in early stages of the pandemic (Salari et al., 2020). This is important to consider since previous research examining longitudinal trajectories of mental health after stressful life events suggests that the majority of the population will adjust to the psychosocial stress arising from the COVID-19 pandemic across time (Chen \& Bonanno, 2020). As such, successful coping will likely be the most common response seen in the general population (Bonanno, 2004). This assumption is broadly consistent with a current meta-analysis of studies comparing mental health before and during the pandemic (Robinson et al., 2021). Notwithstanding, smaller subgroups of individuals will likely develop persistent mental health problems, making the identification of key predictors of early successful coping with COVID-19 an important agenda for current research.

Studies examining successful adjustment to stressful or traumatic life events indicate that flexibility may be a critical contributor to coping with COVID-19 (Chen \& Bonanno, 2020). Broadly speaking, flexibility refers to the ability to employ a variety of emotional, cognitive, 
and behavioral strategies and to adapt them in accordance with changing contextual demands (Cañas et al., 2006; Dennis \& Vander Wal, 2010; Ionescu, 2012; Keith et al., 2015). It enables individuals to shift between discrepant tasks or demands and adapt to different challenges (Koesten et al., 2009; Leung \& Zakzanis, 2014). By emphasizing that successful coping can only be achieved if cognitive, behavioral, and emotional strategies closely match situational demands, the flexibility concept opposes the notion that some strategies are more beneficial that others, rather suggesting that each strategy can have beneficial effects if used in the right situation (Burton \& Bonanno, 2016). The concept of flexibility is thus particularly relevant for coping with multidimensional stressors, which bring about various challenging situations with very diverse characteristics. Such stressors naturally require a repertoire of different strategies that are rapidly deployed in accordance with situational characteristics as well as the ability to flexibly switch between different strategies. Given that the COVID-19 pandemic constitutes an unprecedented multidimensional long-term stressor of the $21^{\text {st }}$ century (Goldfarb, 2020; Gruber et al., 2020), it provides ideal conditions to evaluate how flexibility may tie in with successful coping.

Flexibility can emerge on all levels of intrapsychic processes and has thus been studied in the context of various domains, giving rise to partially overlapping constructs. Two of these constructs that have received substantial attention in the literature are cognitive and regulatory flexibility (Haim-Nachum \& Levy-Gigi, 2021). Cognitive flexibility is the ability to recognize multiple possible responses to a situation and to make an adaptive choice (Martin \& Rubin, 1995). It is thus reflected in a wide repertoire of problem-solving strategies, which are deployed according to situational demands (Kraft et al., 2020). Regulatory flexibility refers to an individual's ability to modulate emotional experience - specifically to up- and downregulate emotional states - using a repertoire of different strategies that match situational demands. Regulatory flexibility can also emerge in the context of complex emotional events such as trauma as reflected in the ability to switch dynamically between engaging with trauma 
by means of reflection and disengaging from trauma by means of focusing on the present (Bonanno et al., 2011). Critically, both cognitive and regulatory flexibility have been linked to reduced psychopathology after trauma (Bonanno et al., 2011; Haim-Nachum \& Levy-Gigi, 2021; Keith et al., 2015; Levy-Gigi et al., 2016; Levy-Gigi et al., 2020), indicating that high flexibility may buffer the potentially deleterious effects of stressful events. A recent study has further shed light on the incremental validity of cognitive flexibility and regulatory flexibility - assessed in the context of emotion expression and coping with trauma - in predicting current and lifetime PTSD symptoms (Haim-Nachum \& Levy-Gigi, 2021). Although all flexibility measures were significantly correlated with PTSD symptoms, cognitive flexibility emerged as the only predictor of current PTSD symptoms with incremental validity. For lifetime symptoms, cognitive and trauma-related regulatory flexibility were found to have incremental validity. Emotion regulatory flexibility was not found to be a unique predictor of either current or lifetime symptoms. These results indicate that - despite significant overlap cognitive and regulatory flexibility seem to encompass unique aspects that are linked to PTSD symptoms.

Although previous findings imply a key role of flexibility in successful coping with trauma, which is informative for identifying potential predictors for coping with the COVID19 pandemic, and the widely held belief that flexibility is particularly critical for coping with multidimensional long-term stressors, only few studies have examined how flexibility may influence coping with the COVID-19 pandemic. First studies investigating the impact of trauma-related regulatory flexibility (Cheng et al., 2021; Jordan et al., 2021; Shigeto et al., 2021) and cognitive flexibility (Kalia et al., 2020; Seiter \& Curran, 2021) on responses to the COVID-19 pandemic revealed that low flexibility is linked to more negative responses. Moreover, two studies found an association between emotion regulatory flexibility and superior mental health in Italian healthcare workers (Lenzo et al., 2021) and elderly outpatients (Sardella et al., 2021) during the COVID-19 pandemic. Critically, no study to date 
has investigated the impact of multiple flexibility constructs (i.e., trauma-related regulatory flexibility, emotion regulatory flexibility, and cognitive flexibility) on coping with the COVID-19 pandemic. Such an investigation seems warranted since multifaceted flexibility is likely required for coping with the COVID-19 pandemic as a multidimensional long-term stressor. Alternatively, high flexibility in one facet might be more important for coping with the pandemic than flexibility in others (Haim-Nachum \& Levy-Gigi, 2021). Moreover, the relevance of these flexibility facets for coping may be modulated by the level of pre-corona stressor exposure. That is, contexts with high levels of stress or trauma exposure may increase requirements for flexible self-regulation whereas these requirements may be lower in contexts with low levels of stress and trauma exposure.

To examine the relevance of multifaceted flexibility as opposed to single flexibility constructs on coping with COVID-19, we conducted a study assessing different flexibility constructs and coping with the COVID-19 pandemic in the general population. To investigate the extent to which result patterns generalize across contexts with high and low pre-corona stressor exposure, we collected subsamples in Israel - being a context of continuous interpersonal trauma exposure - and Germany - being a context without such exposure (Rutter, 1987). During spring and summer 2020, participants from the general population were asked to complete an online study comprising measures of trauma-related regulatory flexibility (Bonanno et al., 2011), cognitive flexibility (Martin \& Rubin, 1995), and emotion regulatory flexibility (Burton \& Bonanno, 2016). Successful coping with the COVID-19 pandemic was assessed on a cognitive, emotional, and behavioral level. Based on these data, we conducted latent profile analyses to investigate the existence of discernable patterns of coping with the COVID-19 pandemic and their association with anxiety and depression symptoms. We tested cross-national similarity of the final class solutions following steps proposed by Morin et al. (2016). Finally, we conducted logistic regression analyses to investigate which facets of flexibility uniquely predict different coping profiles while 
controlling for sociodemographic characteristics and known covariates (Schäfer et al., 2020; Wang et al., 2020; Xu et al., 2020).

\section{Methods}

\section{Participants}

2330 Israelis and 743 Germans participated in the study. Participants were recruited from the general population via online platforms and (social) media advertisements. Recruitment took place from April $12^{\text {th }}$ until May $5^{\text {th }}, 2020$, in Israel and from May $8^{\text {th }}$ until July $1^{\text {st }}, 2020$, in Germany. Those interested in participating were invited to click on a survey link, informing them on the study objectives. They were then asked to provide informed consent. Moreover, they were asked to confirm that they were at least 18 years old and fluent Hebrew or German speakers, respectively. Thereafter, they went on to complete the survey comprising questions on basic demographic variables (e.g., age, gender, and income) and instruments measuring different flexibility constructs, coping with the COVID-19 pandemic, and current levels of depression and anxiety. Finally, lifetime trauma exposure and perceived social support were assessed to serve as covariates. Upon completing the study procedures, participants were thanked for their participation and had the opportunity to enter a raffle. All study procedures were approved by Bar Ilan's Faculty of Education's ethics committee, approval number 65.

\section{Measures}

Assessment of flexibility

Trauma-related regulatory flexibility, was assessed using the Perceived Ability to Cope with Trauma (PACT) scale (Bonanno et al., 2011). The PACT scale measures flexibility in engaging in forward-focused and trauma-focused coping. It comprises 20 items that are rated on a 7-point-scale. Internal consistencies were acceptable in the Israeli (forward focus: $\alpha=$ .86 ; trauma focus: $\alpha=.83$ ) and German (forward focus: $\alpha=.77$; trauma focus: $\alpha=.71$ ) subsamples. In line with Bonanno et al. (2011), PACT items were aggregated by calculating a flexibility score. To this end, the sum of forward-focused and trauma-focused coping items' 
means $($ sum $=$ forward focus + trauma focus) and their absolute polarity (polarity $=[$ forward focus - trauma focus]) were calculated. Then, the polarity score was subtracted from the sum score to obtain the flexibility score (flexibility = sum - polarity).

Cognitive flexibility, was measured using the Cognitive Flexibility Scale (CFS) developed by Martin and Rubin (1995). The CFS conceptualizes cognitive flexibility as a person's awareness that in any given situation there are options and alternatives available, willingness to be flexible and adapt to a certain situation, and self-efficacy in being flexible. The scale comprises 12 items that are rated on a 6-point scale. Previous studies have confirmed the concurrent validity and retest-reliability of the CFS (Martin \& Rubin, 1995). Internal consistencies were good in the current subsamples (Israel: $\alpha=.81$, Germany: $\alpha=.86$ ). A sum score of all items was calculated for the current analyses.

Emotion regulatory flexibility, was measured using the Flexible Regulation of Emotional Expression (FREE) Scale (Burton \& Bonanno, 2016). The FREE assesses an individuals' flexibility in enhancing and suppressing positive and negative emotions across different scenarios. The scale comprises 16 items that are rated on a 6-point-scale. Previous research confirmed the factorial structure of the scale and its discriminant and convergent validity (Burton \& Bonanno, 2016). Internal consistencies were mixed with acceptable internal consistency in the German sample $(\alpha=.76)$ and poor internal consistency in the Israeli sample $(\alpha=.48)$. Based on previous works (Burton \& Bonanno, 2016), a flexibility score was calculated for further analyses. As for the PACT, the sum of enhancing and suppressing items $($ sum $=$ enhancing + suppressing $)$ and their absolute polarity $($ polarity $=[$ enhancing suppressing]) were calculated. Then, the polarity score was subtracted from the sum score to obtain the flexibility score (flexibility $=$ sum - polarity). Assessment of Coping with the COVID-19 Pandemic

Given the lack of tools assessing coping with the COVID-19 pandemic at the time of data collection, we developed a new scale. In developing the scale, we first defined specific facets 
of coping (i.e., emotional, cognitive, and behavioral coping). In a second step, an item pool was developed covering each facet. Of this initial item pool, 9-10 items of each facet were selected for the final scale. Each item was rated on a 5-point-scale. Internal consistency was mostly acceptable in the Israel sample (emotional: $\alpha=.87$, cognitive: $\alpha=.65$, behavioral: $\alpha=$ .55 ) and acceptable in the German sample (emotional: $\alpha=.86$, cognitive: $\alpha=.62$, behavioral: $\alpha=.61)$. Items can be found in Supplementary file A.

\section{Assessment of Depression and Anxiety}

Depression, was assessed using the Beck Depression Inventory (BDI-II) developed by Beck et al. (1996). The BDI-II comprises 21 items that assess symptoms of depression such as hopelessness and irritability, cognitions such as guilt or feelings of being punished, as well as physical symptoms such as fatigue. Internal consistencies in the current sample were high (Israel: $\alpha=.92$, Germany: $\alpha=.94$ ). The sum score of all items was used for the current analyses.

Anxiety, was assessed using the State version of the State-Trait Anxiety Inventory (STAIS) developed by Spielberger (1983). The STAI-S assesses respondents' current anxiety levels using 20 items that are rated on a 4-point scale. Reliability and validity of the scale have been comprehensively investigated and confirmed. In the current sample, internal consistency was found to be high (Israel: $\alpha=.96$, Germany: $\alpha=.96$ ). The sum score of all items was calculated for the current analyses.

\section{Assessment of Potential Covariates}

To examine the effects of Lifetime trauma exposure on coping profiles, we used the Life Events Checklist for DSM-5 (LEC-5) developed by Weathers et al. (2013). The LEC-5 contains sixteen potentially traumatic events. Participants are asked to indicate their level of exposure (happened to me, witnessed it, learned about it, part of my job, not sure or does not apply). In accordance with criterion A of the DSM-5 PTSD criteria (APA, 2013), we calculated the sum of events for which participants indicated direct (happened to me) or 
indirect (witnessed it or part of my job) exposure as our indicator of lifetime trauma exposure.

To assess effects of Social support, we used the Multidimensional Scale of Perceived Social Support (MSPSS) developed by Zimet et al. (1988). The MSPSS consists of 12-items that assess support from friends, from family, and from one's significant other. Each item is rated on a 7-point-scale. Internal consistencies were high in the current sample (Israel: $\alpha=$ .93 , Germany: $\alpha=.94)$. The sum score of all items was calculated for the current analyses.

\section{Data Analysis}

To explore the existence of different coping profiles, we conducted latent profile analyses (LPA) in each subsample using the three coping subscales as indicators. LPA is a mixture modeling technique (McLachlan et al., 2019), which models participants' heterogeneous patterns of responses to latent class indicators as a mix of normal distributions within each class (Peugh \& Fan, 2013). Latent class enumeration determines the number of latent classes and calculates the mean response profile in each latent class (Peugh \& Fan, 2013).

We evaluated models comprising up to five classes. According to widely used methodological recommendations (e.g., Pastor \& Gagné, 2013; Peugh \& Fan, 2013), models were evaluated based on the Akaike's Information Criterion (AIC), the Bayesian Information Criterion (BIC), the sample-size adjusted BIC (SABIC), as well as the adjusted and bootstrapped likelihood ratio test (ALRT and BLRT), and entropy. For AIC, BIC, and SABIC, lower values indicate a model that fits better. ALRT and BLRT test whether a more complex model fits better than a model that has one less latent class. A $p$-value associated with ALRT (BLRT) below the significance level (i.e., .05) indicates a better fit for the more complex model compared to its simpler counterpart. Entropy is an index that quantifies the separation of latent classes and ranges between 0 and 1 , with higher values indicating better class separation. Finally, we also considered parsimony of the model, substantive interpretability of the profiles, and the relative increase in model fit when selecting the final models. LPAs were conducted in Mplus, version 8.6 (Muthén \& Muthén, 2021). In all LPA 
models, means and the variances of the profile indicators in each latent class were estimated freely. The covariances among indicators within latent classes were fixed to zero in all models.

In addition to conducting LPAs within each subsample, we used the approach described by Morin et al. (2016) to assess similarity of profile structure across both countries. To this end, we compared model fits of models imposing configural (number of profiles), structural (within-profile means), dispersion (within-profile means and variability), and distributional (within-profile means, variability and proportions) similarity.

To characterize individual profiles, we compared coping subscales across and within classes. In addition, we investigated differences between coping profiles in depression and anxiety during the pandemic. Lastly, we examined the effects of potential covariates (i.e., sociodemographic variables, social support, and lifetime trauma exposure) and flexibility (i.e., cognitive, emotional, and trauma-related regulatory flexibility) on latent class membership of the best-fitting class solution. We used the three-step approach (multinomial logistic regression) to estimate class membership in relation to these variables while accounting for misspecification bias (Asparouhov \& Muthén, 2014; Vermunt, 2010). The data is available in an online repository: https://osf.io/v8kjm/?view_only=9648976151874ae9ad04d628cfff7ed4

\section{Results}

\section{Baseline Characteristics}

Both subsamples comprised a higher number of female than male participants (Germany: 79.54\%, Israel: 82.59\%), with no significant between-sample differences ( $p$ > $.05)$. Subsamples were found to differ $(p>.05)$ in mean age with German participants $(M=$ $41.32, S D=14.16)$ being younger than Israeli participants $(M=42.69, S D=14.78)$. However, with a mean of 1.37 years, this difference was not substantial. Subsamples did not differ in economic status $(p>.05)$, with the majority of participants in Germany $(M=1.94, S D=0.74)$ 
and Israel $(M=1.99, S D=0.73)$ indicating that their income was around average ${ }^{1}$. Finally, we compared subsamples with respect to trauma history, which did not reveal any significant differences $(p>.05)$ between German $(M=4.19, S D=2.88)$ and Israeli $(M=3.96, S D=3.05)$ participants. To examine differences specifically for interpersonal trauma exposure, we conducted a separate analysis of interpersonal trauma items of the LEC-5 following the criteria proposed by Cloitre et al. (2018). This analysis revealed a significantly higher exposure rate $(p<.001)$ in Israeli $(M=1.54, S D=1.49)$ as compared to German $(M=1.09$, $S D=1.23)$ participants.

\section{Latent Profile Analyses}

In the first step of the LPA, we identified the number of classes that modeled appropriately participants' endorsement of the profile indicators. For the German subsample, $p$-ALRT and p-BLRT became non-significant for the four-class solutions, supporting that a three-class solution was a better fit (see Table 1). Also, the entropy value associated with the three-class solution suggested a better separation of classes than in the four-class solution. For the Israeli subsample $p$-ALRT and $p$-BLRT were significant for the four-class solutions, supporting a four-class solution (see Table 1). However, the entropy value associated with the four-class solution fell below the suggested cut-off of .70. Moreover, closer examination of the fourclass solution revealed that two of four classes were not sufficiently distinct from each other, precluding substantive interpretability of the profiles. By contrast, the three-class solution yielded three clearly differentiable profiles. Thus, based on entropy, parsimony, and substantive interpretability, we selected the three-class solution for the Israeli sample.

Since a three-class solution was selected for each country, configural similarity between countries was supported by the data. Thus, we conducted an LPA across both countries to investigate whether profiles can be interpreted independently of country. Based on this unified

\footnotetext{
${ }^{1} 1$ = below average $/ 2=$ average $/ 3$ =above average $/ 4$ = way above average
} 
analysis, we evaluated whether the configural similarity model - assuming three classes across both countries - had an inferior model fit as compared to the structural similarity model (assuming same class structure as well as equal means), the dispersion similarity model (assuming same class structure as well as equal means and variances) and the distributional similarity model (assuming same class structure as well as equal means, variances and proportions) across countries. This was not the case as reflected in all indicators of model fit (see Table 1). According to Morin et al. (2016), it is thus not viable to interpret results of the LPA analysis unified across both countries. Hence, we continued examining LPAs conducted separately in each subsample. Mean profiles corresponding to the three classes in each subsample are presented in Figure 1 and profiles' characteristics are summarized in Table 2.

In the German sample, a low coping proficiency group comprising $43.20 \%$ of the entire sample. A second group with medium coping proficiency with a tendency towards reduced cognitive coping constituted $40.51 \%$ of the sample, whereas the remaining $16.29 \%$ was characterized by high coping proficiency with a tendency towards enhanced emotional coping. All profiles differed significantly in emotional, cognitive, and behavioral coping.

In the Israeli sample, a low coping proficiency group emerged comprising $21.50 \%$ of the entire sample. A second group with medium coping proficiency with a tendency towards reduced emotional coping constituted $51.20 \%$ of the sample, whereas the remaining $27.30 \%$ was characterized by high coping proficiency with a tendency towards increased emotional coping. All profiles differed significantly in emotional, cognitive, and behavioral coping.

\section{Differences Between Profiles in Depression and Anxiety}

Next, we examined differences among these flexibility profiles regarding depression and anxiety. Means are presented in Table 3. In the German sample, the high coping proficiency group had significantly lower average BDI and STAI-S score than the medium proficiency group, which in turn had significantly lower average scores than the low proficiency group. The average BDI score of the low proficiency group was in the clinically relevant mild-to- 
medium range whereas scores in the medium and high proficiency groups were in the nonclinical range. In the Israeli sample, the high coping proficiency group had significantly lower average BDI and STAI-S score than the medium proficiency group, which in turn had significantly lower average scores than the low proficiency group. The average BDI score of the low proficiency group was in the clinically relevant mild-to-medium range whereas scores in the medium and high proficiency groups were in the non- or sub-clinical range (Table 3).

\section{Predicting Profile Membership Based on Flexibility Facets}

Finally, we used flexibility facets to estimate class membership in multinomial logistic regression analyses. The low proficiency group was used as reference group in both samples. In the German sample, analyses revealed significant effects of gender, social support, lifetime trauma exposure, trauma-related regulatory flexibility, and cognitive flexibility. Accordingly, members of the high coping proficiency group were less likely to be female and more likely to report higher levels of social support and of trauma-related regulatory flexibility as compared to the low proficiency group. Members of the high and average coping proficiency groups were also more like to report lower levels of lifetime trauma exposure and more likely to report higher levels of cognitive flexibility than the low proficiency group. No significant effects emerged for emotion regulatory flexibility (see Table 4).

For the Israeli sample, analyses revealed significant effects of gender, age, economic status, social support, lifetime trauma exposure, trauma-related regulatory flexibility, and cognitive flexibility. The effect of gender reflected that members of the high coping proficiency group were less likely to be female, more likely to report a higher age, higher economic status and emotion regulatory flexibility and lower levels of lifetime trauma exposure than the low proficiency group. Members of the high and average coping proficiency groups were also more likely to report higher levels of social support than the low proficiency group. Finally, members of the high and average coping proficiency group were 
more likely to report higher levels of trauma-related regulatory flexibility and cognitive flexibility than members of the low coping proficiency groups (see Table 5).

\section{Discussion}

The current study aimed to investigate coping during the early stage of the COVID-19 pandemic and how this may be affected by different flexibility domains. To examine crossnational generalizability across conditions of high and low pre-COVID stress, these objectives were assessed in samples of Israeli and German participants. Analyses revealed three distinct coping profiles in each country. Although a test of structural equivalence between countries did not confirm comparable profile structures across countries, there were strong similarities reflected in the emerged profiles (high, medium and low in both countries). The three profiles differed in the severity of anxiety and depression symptoms, with the low coping group reaching clinical symptom levels in both countries. In addition, both cognitive flexibility and trauma-related regulatory flexibility, but not emotion regulatory flexibility, significantly predicted profile membership. Interestingly, however, the relative importance of these predictors differed across countries. That is, in Israel, trauma-related regulatory flexibility was the most important predictor of profile membership, whereas in Germany cognitive flexibility emerged as the most important one.

\section{Characteristics and Distribution of Coping Profiles}

Latent profile analyses conducted separately in each country revealed the existence of three different profiles: A low coping profile, a medium coping profile, and a high coping profile with a tendency towards high emotional coping. In line with the assumption that resilience will be the modal response seen in response to the pandemic (Chen \& Bonanno, 2021), the majority of Israeli (72.7\%) and German (56.8\%) participants showed a high or medium coping profile. Nevertheless, a substantial percentage fell into the low coping profile, further characterized by high state anxiety and clinically significant depression 
symptoms. This finding illustrates the significant impact of the COVID-19 pandemic on the mental health of a vulnerable subpopulation (Robinson et al., 2021; Schäfer et al., 2021).

Due to the lack of structural convergence, it is difficult to draw comparisons between countries based on profile structure. That said, we found that a markedly higher percentage of the German sample than of the Israeli sample was assigned to the low coping profile.

Although this could be related to differences between the characteristics of the low coping profiles in Germany and Israel, with the Israeli sample showing a more extreme low coping profile than the German sample, it could also indicate higher resilience of the Israeli sample during the COVID-19 pandemic. This notion does not align with previous studies comparing mental health responses of German and Israelis during the COVID-19 pandemic, showing comparable levels of distress in both countries (Mækelæ et al., 2020; Mana et al., 2021). However, while the current study had large samples, previous studies had markedly lower sample sizes, especially for Israelis, and assessed indicators of distress rather than coping, which could account for these discrepancies. Notably, research comparing the prevalence of mental health disorders between countries prior to the pandemic found a lower prevalence in Israel (17.6\%) than Germany (25.2\%), supporting the notion of increased resilience (Kessler et al., 2007; Kessler et al., 2010). Amongst other causes, high community resilience and preparedness to cope with sudden national emergencies may relate to our finding of high resilience in the Israeli population (Eshel \& Kimhi, 2016; Leykin et al., 2016). Moreover, Israel's slightly more collectivistic society may act as a protective factor. In support of this assumption, research demonstrates that Asians - coming from a collectivistic culture experience a reduced cumulative impact of previous trauma and COVID-19 related distress as compared to Non-Asians (Ashby et al., 2021). However, since we did not assess any of these potential protective factors, future research is required to test their potential impact on coping during the COVID-19 pandemic. 


\section{Impact of Flexibility on Coping Profiles}

Our regression analyses linking flexibility domains to profile membership, revealed that cognitive flexibility and trauma-related regulatory flexibility significantly predicted membership. In Israel, participants with higher flexibility levels were more likely to be assigned to the medium and high coping group as compared to the low coping group. A similar pattern was evident in the German subsample, with the exception that higher levels of trauma-related regulatory flexibility were only associated with a high probability of being assigned to the high as opposed to the low coping group. This pattern of results aligns with a recent study that examined the incremental contributions of cognitive and regulatory flexibility in predicting current and lifetime PTSD symptoms (Haim-Nachum \& Levy-Gigi, 2021). Cognitive and trauma-related regulatory flexibility were found to be significant predictors, whereas emotion regulatory flexibility was not. Our findings thus replicate previous findings, while additionally expanding them to coping with a multidimensional long-term stressor. As such, they underline the importance of cognitive and trauma-related regulatory flexibility for coping with a range of different stressors (Burton et al., 2012; Palm $\&$ Follette, 2011). Moreover, these flexibility domains were found to have incremental validity after controlling for several important predictors of coping such as economic status, social support, and traumatic exposure.

When comparing the relative contributions of cognitive and trauma-related regulatory flexibility in predicting profile membership, a differential pattern emerged between countries: While trauma-related regulatory flexibility was a stronger predictor than cognitive flexibility in the Israeli sample, cognitive flexibility was a more consistent predictor than trauma-related regulatory flexibility in the German sample. This differential profile of flexibility domains linked to coping with the COVID-19 pandemic, could be related to difference in pre-COVID stress exposure in both countries. That is, trauma-related cognitive flexibility may have been 
of greater importance in the Israeli sample due to the high rate of interpersonal trauma exposure, which provides more opportunities to practice this skill. This may result in individuals developing strong trauma-related flexibility and thus primarily relying on it when coping with various stressors. Since the German population is not systematically subjected to such exposure, there may be a stronger tendency to rely on cognitive as opposed to traumarelated regulatory flexibility. In support of this assumption, Israelis reported a higher number of traumatic events in the interpersonal trauma domain. This is consistent with the types of traumatic events that civilians living in Israel experience (e.g., missile attacks, hostiles throwing stones, stabbing attacks; Bleich et al., 2003; Weinberg et al., 2012). Moreover, when comparing both flexibility domains across countries, a significant interaction between country and flexibility domain emerged, reflecting a stronger difference in trauma-related regulatory flexibility as compared to cognitive flexibility between Israeli and German participants. As expected, Israelis demonstrated markedly higher trauma-related regulatory flexibility scores. However, a smaller, yet significant difference also emerged for the cognitive flexibility. Hence, further longitudinal data is required to readdress whether differences in trauma exposure between countries could be related to differences in the relevance of flexibility domains.

\section{Limitations}

The current study provides new insights on coping processes during the pandemic, yet several limitations must be considered. First and foremost, the current study was crosssectional, which limits us in drawing causal inferences. Future studies should measure flexibility domains and coping continuously, to model longitudinal associations (Schäfer et al., 2021). In addition, we did not use any probabilistic sampling methods, which limit the generalizability of the results to the general population. Relatedly, our sampling strategy resulted in a substantially larger subsample from Israel as compared to Germany and we were 
not able to confirm structural similarity of coping profiles. Nonetheless, both samples were found to be comparable in demographic characteristics. Moreover, result patterns were similar across countries, thus not providing any indications of biased sampling.

Finally, in continuation of our prior research (Haim-Nachum \& Levy-Gigi, 2021), we focused on cognitive and regulatory flexibility rather than assessing other constructs such as behavioral (Brown \& Tait, 2014) or explanatory flexibility (Joseph \& Gray, 2011). Future studies should consider assessing these constructs in order to provide a more comprehensive view on the incremental validity of individual flexibility domains.

\section{Outlook}

Overall, the current findings support previous research in showing that the COVID-19 pandemic is a significant stressor that impacts the mental health of a subgroup of the population. Moreover, in line with previous research, we found that cognitive and traumarelated regulatory flexibility were linked to successful coping with the pandemic, thus establishing these flexibility domains as predictors of coping with a multidimensional - not necessarily traumatic - stressor. If replicated by future longitudinal studies, these findings may have important practical implications. They suggest that training cognitive and traumarelated regulatory flexibility may help individuals to better cope with long-term stressors such as the COVID-19 pandemic. Such trainings could be selectively administered to a subpopulation with low levels of flexibility in these domains. Critically, our results suggest that it may be useful to adapt such trainings to the specifics of the respective population. That is, a stronger focus may be laid on either cognitive or trauma-related regulatory flexibility based on the flexibility profile that is found to be beneficial in the respective population. 


\section{References}

APA. (2013). Diagnostic and statistical manual of mental disorders (DSM-5®). American Psychiatric Pub.

Ashby, J. S., Rice, K. G., Kira, I. A., \& Davari, J. (2021). The relationship of COVID-19 traumatic stress, cumulative trauma, and race to posttraumatic stress disorder symptoms. Journal of Community Psychology.

Asparouhov, T., \& Muthén, B. (2014). Auxiliary variables in mixture modeling: Three-step approaches using M plus. Structural Equation Modeling: A Multidisciplinary Journal, 21(3), 329-341.

Beck, A. T., Steer, R. A., \& Brown, G. (1996). Beck depression inventory-II. Psychological Assessment.

Bleich, A., Gelkopf, M., \& Solomon, Z. (2003). Exposure to terrorism, stress-related mental health symptoms, and coping behaviors among a nationally representative sample in Israel. Jama, 290(5), 612-620.

Bonanno, G. A. (2004). Loss, trauma, and human resilience: Have we underestimated the human capacity to thrive after extremely aversive events? American Psychologist, 59(1), 20-28.

Bonanno, G. A., Pat-Horenczyk, R., \& Noll, J. (2011). Coping flexibility and trauma: The Perceived Ability to Cope with Trauma (PACT) scale. Psychological Trauma: Theory, Research, Practice, and Policy, 3(2), 117-129.

Brown, V. J., \& Tait, D. S. (2014). Behavioral flexibility: Attentional shifting, rule switching and response reversal. Encyclopedia of Psychopharmacology, 1-7.

Burton, C. L., \& Bonanno, G. A. (2016). Measuring ability to enhance and suppress emotional expression: The Flexible Regulation of Emotional Expression (FREE) scale. Psychological Assessment, 28(8), 929-941. 
Burton, C. L., Yan, O. H., Pat-Horenczyk, R., Chan, I. S., Ho, S., \& Bonanno, G. A. (2012). Coping flexibility and complicated grief: A comparison of American and Chinese samples. Depression and Anxiety, 29(1), 16-22.

Cañas, J. J., Fajardo, I., \& Salmeron, L. (2006). Cognitive flexibility. International Encyclopedia of Ergonomics and Human Factors, 1, 297-301.

Chen, S., \& Bonanno, G. A. (2020). Psychological adjustment during the global outbreak of COVID-19: A resilience perspective. Psychological Trauma: Theory, Research, Practice, and Policy, 12(S1), S51.

Cheng, C., Wang, H.-Y., \& Ebrahimi, O. V. (2021). Adjustment to a "new normal": Mental health benefits of coping flexibility during the COVID-19 pandemic. Frontiers in Psychiatry, 12, 353.

Cloitre, M., Shevlin, M., Brewin, C. R., Bisson, J. I., Roberts, N. P., Maercker, A., Karatzias, T., \& Hyland, P. (2018). The International Trauma Questionnaire: Development of a self-report measure of ICD-11 PTSD and complex PTSD. Acta Psychiatrica Scandinavica, 138(6), 536-546.

Dennis, J. P., \& Vander Wal, J. S. (2010). The cognitive flexibility inventory: Instrument development and estimates of reliability and validity. Cognitive Therapy and Research, 34(3), 241-253.

Eshel, Y., \& Kimhi, S. (2016). Community resilience of civilians at war: A new perspective. Community Mental Health Journal, 52(1), 109-117.

Goldfarb, E. V. (2020). Participant stress in the COVID-19 era and beyond. Nature Reviews Neuroscience, 21(12), 663-664.

Gruber, J., Prinstein, M. J., Clark, L. A., Rottenberg, J., Abramowitz, J. S., Albano, A. M., Aldao, A., Borelli, J. L., Chung, T., \& Davila, J. (2020). Mental health and clinical 
psychological science in the time of COVID-19: Challenges, opportunities, and a call to action. American Psychologist.

Haim-Nachum, S., \& Levy-Gigi, E. (2021). The tension between cognitive and regulatory flexibility and their associations with current and lifetime PTSD symptoms [brief research report]. Frontiers in Psychology, 12(361).

Ionescu, T. (2012). Exploring the nature of cognitive flexibility. New Ideas in Psychology, 30(2), 190-200.

Jordan, L. S., Woodard, J. L., Pena, G. S., Arnold-Nedimala, N. A., Won, J., Callow, D. D., \& Smith, J. C. (2021). Forward-focused coping predicts better mental health outcomes in mid-to late-life during the COVID-19 pandemic. Aging and Mental Health, 1-9.

Joseph, J. S., \& Gray, M. J. (2011). The utility of measuring explanatory flexibility in PTSD research. Cognitive Therapy and Research, 35(4), 372-380.

Kalia, V., Knauft, K., \& Hayatbini, N. (2020). Cognitive flexibility and perceived threat from COVID-19 mediate the relationship between childhood maltreatment and state anxiety. PLoS ONE, 15(12), e0243881.

Keith, J., Velezmoro, R., \& O’Brien, C. (2015). Correlates of cognitive flexibility in veterans seeking treatment for posttraumatic stress disorder. The Journal of Nervous and Mental Disease, 203(4), 287-293.

Kessler, R. C., Angermeyer, M., Anthony, J. C., De Graaf, R. O. N., Demyttenaere, K., Gasquet, I., ... \& Uestuen, T. B. (2007). Lifetime prevalence and age-of-onset distributions of mental disorders in the World Health Organization's World Mental Health Survey Initiative. World Psychiatry, 6(3), 168-176.

Kessler, R. C., McLaughlin, K. A., Green, J. G., Gruber, M. J., Sampson, N. A., ... \& Williams, D. R. (2010). Childhood adversities and adult psychopathology in the WHO World Mental Health Surveys. The British Journal of Psychiatry, 197(5), 378-385. 
Koesten, J., Schrodt, P., \& Ford, D. J. (2009). Cognitive flexibility as a mediator of family communication environments and young adults' well-being. Health Communication, 24(1), 82-94.

Kraft, D., Rademacher, L., Eckart, C., \& Fiebach, C. J. (2020). Cognitive, Affective, and Feedback-Based Flexibility - Disentangling Shared and Different Aspects of Three Facets of Psychological Flexibility. Journal of Cognition, 3(1), 21.

Krishnamoorthy, Y., Nagarajan, R., Saya, G. K., \& Menon, V. (2020). Prevalence of psychological morbidities among general population, healthcare workers and COVID19 patients amidst the COVID-19 pandemic: A systematic review and meta-analysis. Psychiatry Research, 293, 113382.

Lenzo, V., Quattropani, M. C., Sardella, A., Martino, G., \& Bonanno, G. A. (2021). Depression, Anxiety, and Stress Among Healthcare Workers During the COVID-19 Outbreak and Relationships With Expressive Flexibility and Context Sensitivity. Frontiers in Psychology, 12, 348.

Leung, R. C., \& Zakzanis, K. K. (2014). Brief report: cognitive flexibility in autism spectrum disorders: a quantitative review. Journal of Autism and Developmental Disorders, 44(10), 2628-2645.

Levy-Gigi, E., Donner, R., \& Bonanno, G. A. (2020). Free Your Mind: Emotional Expressive Flexibility Moderates the Effect of Stress on Post-Traumatic Stress Disorder Symptoms. International Journal of Molecular Sciences, 21(15), 5355.

Levy-Gigi, E., Bonanno, G. A., Shapiro, A. R., Richter-Levin, G., Kéri, S., \& Sheppes, G. (2016). Emotion regulatory flexibility sheds light on the elusive relationship between repeated traumatic exposure and posttraumatic stress disorder symptoms. Clinical Psychological Science, 4(1), 28-39. 
Leykin, D., Lahad, M., Cohen, R., Goldberg, A., \& Aharonson-Daniel, L. (2016). The dynamics of community resilience between routine and emergency situations. International Journal of Disaster Risk Reduction, 15(1), 125-131.

Mækelæ, M. J., Reggev, N., Dutra, N., Tamayo, R. M., Silva-Sobrinho, R. A., Klevjer, K., \& Pfuhl, G. (2020). Perceived efficacy of COVID-19 restrictions, reactions and their impact on mental health during the early phase of the outbreak in six countries. Royal Society Open Science, 7(8), 200644.

Mana, A., Bauer, G., Magistretti, C. M., Sardu, C., Juvinyà-Canal, D., Hardy, L., Catz, O., Tušl, M., \& Sagy, S. (2021). Order out of Chaos: Sense of coherence and the mediating role of coping resources in explaining mental health during COVID-19 in 7 countries. SSM-Mental Health, 1, 100001.

Martin, M. M., \& Rubin, R. B. (1995). A new measure of cognitive flexibility. Psychological Reports, 76(2), 623-626.

McLachlan, G. J., Lee, S. X., \& Rathnayake, S. I. (2019). Finite mixture models. Annual Review of Statistics and its Application, 6, 355-378.

Morin, A. J., Meyer, J. P., Creusier, J., \& Biétry, F. (2016). Multiple-group analysis of similarity in latent profile solutions. Organizational research methods, 19(2), 231-254.

Palm, K. M., \& Follette, V. M. (2011). The roles of cognitive flexibility and experiential avoidance in explaining psychological distress in survivors of interpersonal victimization. Journal of Psychopathology and Behavioral Assessment, 33(1), 79-86.

Paredes, M. R., Apaolaza, V., Fernandez-Robin, C., Hartmann, P., \& Yañez-Martinez, D. (2021). The impact of the COVID-19 pandemic on subjective mental well-being: The interplay of perceived threat, future anxiety and resilience. Personality and Individual Differences, $170,110455$.

Pastor, D. A., \& Gagné, P. (2013). Mean and covariance structure mixture models. 
Peugh, J., \& Fan, X. (2013). Modeling unobserved heterogeneity using latent profile analysis: A Monte Carlo simulation. Structural Equation Modeling: A Multidisciplinary Journal, 20(4), 616-639.

Pfefferbaum, B., \& North, C. S. (2020). Mental health and the Covid-19 pandemic. New EnglandJjournal of Medicine, 383(6), 510-512.

Robinson, E., Sutin, A. R., Daly, M., \& Jones, A. (2021). A systematic review and metaanalysis of longitudinal cohort studies comparing mental health before versus during the COVID-19 pandemic. medRxiv.

Rutter, M. (1987). Psychosocial resilience and protective mechanisms. American Journal of Orthopsychiatry, 57(3), 316-331.

Salari, N., Hosseinian-Far, A., Jalali, R., Vaisi-Raygani, A., Rasoulpoor, S., Mohammadi, M., Rasoulpoor, S., \& Khaledi-Paveh, B. (2020). Prevalence of stress, anxiety, depression among the general population during the COVID-19 pandemic: a systematic review and meta-analysis. Globalization and Health, 16(1), 1-11.

Sardella, A., Lenzo, V., Bonanno, G. A., Basile, G., \& Quattropani, M. C. (2021). Expressive flexibility and dispositional optimism contribute to the elderly's resilience and healthrelated quality of life during the COVID-19 pandemic. International Journal of Environmental Research and Public Health, 18(4), 1698.

Schäfer, S., Sopp, R., Koch, M., Göritz, A. S., \& Michael, T. (2021). The long-term buffering effect of sense of coherence on psychopathological symptoms during the COVID-19 pandemic: Findings from a prospective observational study.

Schäfer, S. K., Sopp, M. R., Schanz, C. G., Staginnus, M., Göritz, A. S., \& Michael, T. (2020). Impact of COVID-19 on public mental health and the buffering effect of a sense of coherence. Psychotherapy and Psychosomatics. 
Seiter, J. S., \& Curran, T. (2021). Social-distancing fatigue during the COVID-19 pandemic: a mediation analysis of cognitive flexibility, fatigue, depression, and adherence to CDC guidelines. Communication Research Reports, 38(1), 68-78.

Shigeto, A., Laxman, D. J., Landy, J. F., \& Scheier, L. M. (2021). Typologies of coping in young adults in the context of the COVID-19 pandemic. The Journal of General Psychology, 1-34.

Spielberger, C. D. (1983). State-trait anxiety inventory for adults.

Vermunt, J. K. (2010). Latent class modeling with covariates: Two improved three-step approaches. Political Analysis, 18(4), 450-469.

Wang, Y., Kala, M. P., \& Jafar, T. H. (2020). Factors associated with psychological distress during the coronavirus disease 2019 (COVID-19) pandemic on the predominantly general population: A systematic review and meta-analysis. PLOS ONE, 15(12), $\mathrm{e} 0244630$.

Weathers, F., Blake, D., Schnurr, P., Kaloupek, D., Marx, B., \& Keane, T. (2013). The life events checklist for DSM-5 (LEC-5).

Weinberg, M., Besser, A., Campeas, M., Shvil, E., \& Neria, Y. (2012). Reactions of civilians exposed to terrorism and war trauma in Israel: The role of intra- and interpersonal factors. In A. M. Columbus (Ed.), Advances in psychology research (pp. 1-53). Nova Science Publishers.

Xu, J. J., Ou, J. Y., Luo, S. Y., Wang, Z. J., Chang, E., Novak, C., Shen, J. Y., Zheng, S. Y., \& Wang, Y. A. (2020). Perceived social support protects lonely people against COVID19 anxiety: A three-wave longitudinal study in China. Frontiers in Psychology, 11, 66965.

Zimet, G. D., Dahlem, N. W., Zimet, S. G., \& Farley, G. K. (1988). The multidimensional scale of perceived social support. Journal of Personality Assessment, 52(1), 30-41. 


\section{Table 1}

Statistical Criteria Associated with Latent Class Enumeration.

\begin{tabular}{|c|c|c|c|c|c|c|c|c|c|}
\hline Model & $\mathrm{k}$ & SSS & LL & AIC & $\mathrm{BIC}$ & SABIC & $p$-ALRT & $p$-BLRT & Entropy \\
\hline \multicolumn{10}{|c|}{ Class Enumeration: Germany } \\
\hline 1-class & 1 & - & -7522.27 & 15056.54 & 15084.21 & 15065.15 & - & - & - \\
\hline 2-class & 2 & 339 & -7224.69 & 14469.39 & 14515.50 & 14483.74 & $<.001$ & $<.001$ & 0.78 \\
\hline 3-class & 3 & 121 & -7170.21 & 14368.42 & 14432.97 & 14388.52 & .003 & .003 & 0.74 \\
\hline 4-class & 4 & 50 & -7157.90 & 14351.80 & 14434.79 & 14377.63 & .519 & .528 & 0.69 \\
\hline \multicolumn{10}{|c|}{ Class Enumeration: Israel } \\
\hline 1-class & 1 & - & -23174.58 & 46361.15 & 46395.67 & 46376.61 & - & - & - \\
\hline 2-class & 2 & 933 & -22441.40 & 44902.81 & 44960.35 & 44928.57 & $<.001$ & $<.001$ & 0.73 \\
\hline 3-class & 3 & 501 & -22243.22 & 44514.43 & 44594.98 & 44550.50 & $<.001$ & $<.001$ & 0.70 \\
\hline 4-class & 4 & 254 & -22183.53 & 44403.05 & 44506.62 & 44449.43 & $<.001$ & $<.001$ & 0.67 \\
\hline \multicolumn{10}{|c|}{ Cross-national Similarity } \\
\hline Configural & 3 & 121 & -14035.91 & 28153.82 & 28367.77 & 28237.53 & - & - & 0.69 \\
\hline Structural & 3 & 121 & -14069.31 & 28202.61 & 28369.60 & 28267.94 & - & - & 0.68 \\
\hline Dispersion & 3 & 121 & -14105.47 & 28244.93 & 28333.64 & 28279.64 & - & - & 0.67 \\
\hline Distributional & 3 & 121 & -14110.61 & 28257.23 & 28351.16 & 28293.98 & - & - & 0.67 \\
\hline
\end{tabular}

Note. $\mathrm{SSS}=$ the smallest sample size associated with any of the classes identified by the given model; $\mathrm{LL}=$ log-likelihood $; \mathrm{AIC}=$ Akaike Information Criterion; $\mathrm{BIC}=$ Bayesian Information Criterion; SABIC $=$ sample-size adjusted BIC; $p$-ALRT $=$ the $p$-value associated with the adjusted likelihood ratio test; $p$-BLRT $=$ the $p$-value associated with the bootstrapped likelihood ratio test. 


\section{Table 2}

Characteristics of individual profiles

\begin{tabular}{|c|c|c|c|c|c|c|c|c|c|}
\hline \multirow[t]{3}{*}{ Country } & \multirow[t]{3}{*}{ Latent class } & \multirow[t]{3}{*}{$\mathrm{n}$} & \multicolumn{2}{|c|}{ Emotional } & \multicolumn{2}{|c|}{ Cognitive } & \multicolumn{2}{|c|}{ Behavioral } & \multirow{3}{*}{$\begin{array}{l}\text { Significant } \\
\text { differences }(\mathrm{p}< \\
.05)\end{array}$} \\
\hline & & & M & SD & M & SD & M & SD & \\
\hline & & & & & & & & & \\
\hline \multirow[t]{3}{*}{ Germany } & Profile 1 & 321 & -0.79 & 0.50 & -0.75 & 0.79 & -0.79 & 0.76 & $\mathrm{C}, \mathrm{B}, \mathrm{E}$ \\
\hline & Profile 2 & 301 & 0.54 & 0.50 & 0.00 & 0.83 & 0.47 & 0.68 & $\mathrm{E}, \mathrm{B}>\mathrm{C}$ \\
\hline & Profile 3 & 121 & 1.73 & 0.49 & 0.99 & 0.84 & 1.25 & 0.63 & $E>B>C$ \\
\hline \multirow[t]{3}{*}{ Israel } & Profile 1 & 501 & -1.10 & 0.42 & -0.92 & 0.72 & -1.24 & 0.69 & $C>E>B$ \\
\hline & Profile 2 & 1193 & -0.27 & 0.57 & 0.01 & 0.74 & 0.04 & 0.65 & $\mathrm{~B}, \mathrm{C}>\mathrm{E}$ \\
\hline & Profile 3 & 636 & 1.19 & 0.52 & 0.91 & 0.80 & 0.83 & 0.67 & $\mathrm{E}>\mathrm{C}, \mathrm{B}$ \\
\hline
\end{tabular}

Note. The table presents standardized z-scores for easier interpretation. Profiles differed significantly on all coping scales. Germany - Emotional coping: $\mathrm{P} 1<\mathrm{P} 2$ : $\mathrm{T}(620)=33.27$, $\mathrm{p}<$ $.001 ; \mathrm{P} 2<\mathrm{P} 3: \mathrm{T}(420)=21.97, \mathrm{p}<.001 ; \mathrm{P} 1<\mathrm{P} 3: \mathrm{T}(440)=47.37, \mathrm{p}<.001$; Cognitive coping: $\mathrm{P} 1<\mathrm{P} 2: \mathrm{T}(620)=11.57, \mathrm{p}<.001 ; \mathrm{P} 2<\mathrm{P} 3: \mathrm{T}(420)=10.94, \mathrm{p}<.001 \mathrm{P} 1<\mathrm{P} 3: \mathrm{T}(440)=$ 20.23, $\mathrm{p}<.001$; Behavioral Coping: $\mathrm{P} 1<\mathrm{P} 2: \mathrm{T}(620)=21.81, \mathrm{p}<.001 ; \mathrm{P} 2<\mathrm{P} 3: \mathrm{T}(420)=$ 10.87, $\mathrm{p}<.001 ; \mathrm{P} 1<\mathrm{P} 3: \mathrm{T}(440)=26.30, \mathrm{p}<.001$. Israel - Emotional coping: $\mathrm{P} 1<\mathrm{P} 2$ : $\mathrm{T}(1692)=29.66, \mathrm{p}<.001 ; \mathrm{P} 2<\mathrm{P} 3: \mathrm{T}(1827)=53.79, \mathrm{p}<.001 ; \mathrm{P} 1<\mathrm{P} 3: \mathrm{T}(1135)=79.98, \mathrm{p}<$ .001; Cognitive coping: P1 < P2: T(1692) = 23.73, p <.001; $\mathrm{P} 2<\mathrm{P} 3: \mathrm{T}(1827)=24.02, \mathrm{p}<$ $.001 \mathrm{P} 1<\mathrm{P} 3: \mathrm{T}(1135)=39.95, \mathrm{p}<.001 ;$ Behavioral Coping: $\mathrm{P} 1<\mathrm{P} 2: \mathrm{T}(1692)=36.31, \mathrm{p}<$ $.001 ; \mathrm{P} 2<\mathrm{P} 3: \mathrm{T}(1827)=24.46, \mathrm{p}<.001 ; \mathrm{P} 1<\mathrm{P} 3: \mathrm{T}(1135)=50.97, \mathrm{p}<.001$. 


\section{Table 3}

Comparing depression and anxiety as predicted by profiles in each country.

\begin{tabular}{lllllllll}
\hline Country & Outcome & Profile 1 & \multicolumn{5}{c}{ Profile 2 } & Profile 3 \\
& & $\mathrm{M}$ & $\mathrm{SE}$ & $\mathrm{M}$ & $\mathrm{SE}$ & $\mathrm{M}$ & $\mathrm{SE}$ & Significant differences \\
& & & & & & & & $(\mathrm{p}<.001)$ \\
\hline Germany & Depression & 20.65 & 0.67 & 8.28 & 0.55 & 2.89 & 0.58 & $\mathrm{P} 1>\mathrm{P} 2>\mathrm{P} 3$ \\
& Anxiety & 57.48 & 0.71 & 39.58 & 0.69 & 28.70 & 0.93 & $\mathrm{P} 1>\mathrm{P} 2>\mathrm{P} 3$ \\
\multirow{2}{*}{ Israel } & Depression & 22.72 & 0.64 & 11.28 & 0.31 & 4.32 & 0.24 & $\mathrm{P} 1>\mathrm{P} 2>\mathrm{P} 3$ \\
& Anxiety & 61.09 & 0.64 & 43.84 & 0.42 & 30.62 & 0.41 & $\mathrm{P} 1>\mathrm{P} 2>\mathrm{P} 3$ \\
& & & & & & & & \\
\end{tabular}

Note. Germany - BDI: P1 > P2: $\chi=180.52, \mathrm{p}<.001 ; \mathrm{P} 2>\mathrm{P} 3: \chi=36.00, \mathrm{p}<.001 ; \mathrm{P} 1>\mathrm{P} 3: \chi=$ 411.07, $<$ <.001; STAI: P1 > P2: $\chi=285.11, \mathrm{p}<.001 ; \mathrm{P} 2>\mathrm{P} 3: \chi=71.78, \mathrm{p}<.001 ; \mathrm{P} 1>\mathrm{P} 3:$ $\chi=603.00, p<.001$; Israel - BDI: P1 > P2: $\chi=217.33, p<.001 ;$ P2 > P3: $\chi=262.04, p<$ $.001 ; \mathrm{P} 1>\mathrm{P} 3: \chi=748.56, \mathrm{p}<.001$; STAI: P1 > P2: $\chi=429.80, \mathrm{p}<.001 ; \mathrm{P} 2>\mathrm{P} 3: \chi=431.59, \mathrm{p}$ $<.001 ; \mathrm{P} 1>\mathrm{P} 3: \chi=1617.22, \mathrm{p}<.001$ 


\section{Table 4}

Predicting profiles based on sociodemographic variables, social support, lifetime trauma exposure, and flexibility facets in the German subsample

\begin{tabular}{|c|c|c|c|c|c|c|}
\hline \multirow[t]{2}{*}{ Predictor } & \multicolumn{3}{|c|}{ Profile 2} & \multicolumn{3}{|l|}{ Profile 3} \\
\hline & B & OR & $95 \%$ & B & OR & $95 \%$ \\
\hline Age & -0.01 & 0.99 & $0.98-1.00$ & 0.00 & 1.00 & $0.97-1.02$ \\
\hline Gender & -0.46 & 0.63 & $0.33-1.20$ & $-1.49^{* * *}$ & 0.23 & $0.11-0.48$ \\
\hline Economic status & 0.21 & 1.23 & $0.90-1.69$ & 0.28 & 1.32 & $0.83-2.09$ \\
\hline Social support & 0.01 & 1.01 & $1.00-1.02$ & $0.06^{* *}$ & 1.06 & $1.01-1.10$ \\
\hline Lifetime Trauma exposure & $-0.10^{*}$ & 0.91 & $0.83-0.98$ & $-0.24^{* * *}$ & 0.79 & $0.70-0.90$ \\
\hline Emotion regulatory flexibility & 0.01 & 1.01 & $0.99-1.03$ & -0.01 & 0.99 & $0.97-1.02$ \\
\hline Trauma-related regulatory & 0.04 & 1.04 & $0.93-1.16$ & $0.28^{* * *}$ & 1.32 & $1.13-1.56$ \\
\hline \multicolumn{7}{|l|}{ flexibility } \\
\hline Cognitive flexibility & $0.08^{* * *}$ & 1.09 & $1.05-1.12$ & $0.16^{* * *}$ & 1.17 & $1.11-1.24$ \\
\hline
\end{tabular}

Note. ${ }^{*} p<.05,{ }^{* *} p<.01,{ }^{* * *} p<.001 ; \mathrm{OR}=$ odds ratio, Reference $=$ Profile 1 


\section{Table 5}

Predicting profiles based on sociodemographic variables, social support, lifetime trauma exposure, and flexibility facets in the Israeli subsample

\begin{tabular}{llllllll}
\hline Predictor & Profile 2 & & \multicolumn{5}{l}{ Profile 3} \\
& B & OR & $95 \%$ & B & OR & $95 \%$ \\
\hline Age & 0.01 & 1.01 & $1.00-1.02$ & $0.04^{* * *}$ & 1.04 & $1.03-1.05$ \\
Gender & -0.25 & 0.78 & $0.51-1.21$ & $-0.66^{* *}$ & 0.52 & $0.33-0.80$ \\
Economic status & 0.15 & 1.16 & $0.91-1.45$ & $0.53^{* * *}$ & 1.71 & $1.33-2.19$ \\
Social support & $0.03^{* * *}$ & 1.03 & $1.02-1.04$ & $0.04^{* * *}$ & 1.04 & $1.03-1.06$ \\
Lifetime Trauma exposure & -0.04 & 0.97 & $0.92-1.02$ & $-0.11^{* * *}$ & 0.90 & $0.85-0.95$ \\
Emotion regulatory flexibility & 0.01 & 1.01 & $0.99-1.02$ & $0.02^{*}$ & 1.02 & $1.00-1.04$ \\
Trauma-related regulatory & $0.18^{* * *}$ & 1.20 & $1.09-1.31$ & $0.34^{* * *}$ & 1.40 & $1.27-1.55$ \\
flexibility & & & & & & \\
Cognitive flexibility & $0.03^{* *}$ & 1.03 & $1.01-1.06$ & $0.06^{* * *}$ & 1.06 & $1.03-1.09$ \\
\hline
\end{tabular}

$\overline{\text { Note } .}{ }^{*} p<.05,{ }^{* *} p<.01,{ }^{* * *} p<.001 ; \mathrm{OR}=$ odds ratio, $p$-ALRT $=$ the $\mathrm{p}$-value associated $w i t h$ the adjusted likelihood ratio test, Reference $=$ Profile 1 . 